\title{
Quantum Brownian motion and its conflict with the second law
}

\author{
Theo M. Nieuwenhuizen* and Armen E. Allahverdyan ${ }^{\dagger}$ \\ *Institute for Theoretical Physics, Valckenierstraat 65, 1018 XE Amsterdam, The Netherlands \\ ${ }^{\dagger}$ Yerevan Physics Institute, Alikhanian Brothers St. 2, Yerevan 375036, Armenia
}

\begin{abstract}
.
The Brownian motion of a harmonically bound quantum particle and coupled to a harmonic quantum bath is exactly solvable. At low enough temperatures the stationary state is non-Gibbsian due to an entanglement with the bath. This happens when a cloud of bath modes around the particle is formed. Equilibrium thermodynamics for particle plus bath together, does not imply standard thermodynamics for the particle itself at low $T$. Various formulations of the second law are then invalid. First, the Clausius inequality can be violated. Second, when the width of the confining potential is suddenly changed, there occurs a relaxation to equilibrium during which the rate of entropy production is partly negative. Third, for non-adiabatic changes of system parameters the rate of energy dissipation can be negative, and, out of equilibrium, cyclic processes are possible which extract work from the bath. Conditions are put forward under which perpetuum mobile of the second kind, having several work extraction cycles, enter the realm of condensed matter physics.
\end{abstract}

Introduction. There are not two fundamental theories of nature, quantum mechanics and thermodynamics. There is only one: quantum mechanics, while thermodynamics must emerge from it. The universal character of equilibrium thermodynamics led to the general expectation that in one way or another, thermodynamics will apply to the full quantum domain [1]. Few people have taken the painful road to check this emergence, yet this is what we have set out to do. Here we discuss the results for quantum Brownian motion that have been presented [2, 3] and were discussed in the scientific literature [\#].

Brownian motion has numerous applications in condensed matter physics [5, 6, 7, 8], atomic physics [5], quantum optics and chemistry [9]. Some realizations involve weak coupling with the thermal bath [9]. However, there are well-known experimental situations, which are essentially far from the weak-coupling regime. Here standard thermodynamics may not apply. The main example of this is the case of weak links between superconductive regions, the so-called Josephson junctions, in their overdamped regime [10, 11], where the relevant ranges of parameters were achieved already twenty years ago. Even in quantum optics, which has often been satisfactorily described by weakcoupling theories [9], there are recent experiments showing the necessity for moderate and strong coupling approaches [12].

The Hamiltonian. We consider an 'ideal gas' of non-interacting harmonic oscillators coupled to a bath. For the total Hamiltonian $\mathscr{H}_{\text {tot }}=\mathscr{H}+\mathscr{H}_{B}+\mathscr{H}_{I}$ we thus assume [6]

$\mathscr{H}=\frac{p^{2}}{2 m}+\frac{1}{2} a x^{2}, \quad \mathscr{H}_{B}=\sum_{i}\left[\frac{p_{i}^{2}}{2 m_{i}}+\frac{m_{i} \omega_{i}^{2}}{2} x_{i}^{2}\right], \quad \mathscr{H}_{I}=\sum_{i}\left[-c_{i} x_{i} x+\frac{c_{i}^{2}}{2 m_{i} \omega_{i}^{2}} x^{2}\right]$, 
with $\mathscr{H}$ describing the particle, $\mathscr{H}_{B}$ the bath, and $\mathscr{H}_{I}$ the interaction.

The bath is assumed to have uniformly spaced modes $\omega_{i}=i \Delta, \quad i=1,2,3, \cdots$. The thermodynamic limit for the bath is taken by sending $\Delta \rightarrow 0$, which induces relaxational behavior. For the couplings we choose the quasi-Ohmic Drude-Ullersma spectrum, where [6] $J(\omega)=\frac{1}{2} \pi \sum_{i}\left(c_{i}^{2} / m_{i} \omega_{i}\right) \delta\left(\omega-\omega_{i}\right)=\gamma \omega \Gamma^{2} /\left(\omega^{2}+\Gamma^{2}\right)$, where $\gamma$ is the damping constant. We shall assume that the Debye cutoff $\Gamma$ is large.

We describe an ensemble of closed total systems with conserved energy, except for the periods when work is done on it by externally changing $m$ or $a$. We take as initial density matrix the Gibbs distribution $\exp \left(-\beta \mathscr{H}_{\text {tot }}\right) / Z_{\text {tot }}$, with $Z_{\text {tot }}$ the partition sum.

Equilibrium state. In Gibbsian equilibrium of the total system the free energy reads $F_{\text {tot }}(T, \gamma)=F_{B}(T, \gamma=0)+F_{p}(a, \gamma, \Gamma, m, T)$. The free energy of the bath itself,

$$
F_{B}(T, \gamma=0)=\frac{T}{\Delta} \int_{0}^{\infty} \mathrm{d} \omega \ln \left(1-e^{-\beta \hbar \omega}\right)=-\frac{\pi^{2} T^{2}}{6 \hbar \Delta}
$$

is of order $1 / \Delta$, showing the extensivity of the bath. The Brownian particle adds to this

$$
F_{p}=T\left[\ln \Gamma\left(\frac{\beta \hbar \Gamma}{2 \pi}\right)-\ln \Gamma\left(\frac{\beta \hbar \omega_{1}}{2 \pi}\right)-\ln \Gamma\left(\frac{\beta \hbar \omega_{2}}{2 \pi}\right)-\ln \Gamma\left(\frac{\beta \hbar \omega_{3}}{2 \pi}\right)-\ln \frac{\beta \hbar \omega_{0}}{(2 \pi)^{2}}\right] .
$$

It contains three characteristic, temperature independent of frequencies. For small $\gamma$ (underdamping) they read: $\omega_{1,2}= \pm i \omega_{0}+\gamma / 2 m, \omega_{3}=\Gamma-\gamma / m$. On the other hand, for strong damping $\gamma^{2} \gg a m, \omega_{1}=a / \gamma, \omega_{2}=(\gamma / m)\left(1-a m / \gamma^{2}\right), \omega_{3}=\Gamma-\gamma / m$.

Effective temperatures. We shall now study two objects, $T_{x}=a\left\langle x^{2}\right\rangle$ and $T_{p}=\left\langle p^{2}\right\rangle / m$, that would in classical equilibrium be equal to $T$ and which we shall interpret below as effective temperatures. As in the classical situation, it holds that $T_{x}=2 a \partial F_{p} / \partial a$, $T_{p}=-2 m \partial F_{p} / \partial m$. For $\gamma \rightarrow 0$ one gets the weak-coupling result known from textbooks, $U \equiv \frac{1}{2} T_{x}+\frac{1}{2} T_{p}=T_{x}=T_{p}=\frac{1}{2} \hbar \omega_{0} \operatorname{coth} \frac{1}{2} \beta \hbar \omega_{0}$, with $\omega_{0}=\sqrt{a / m}$. At large $T$ one gets

$$
T_{x}=T+\frac{\beta \hbar^{2} a}{12 m}, \quad T_{p}=T+\frac{\beta \hbar^{2}(a+\gamma \Gamma)}{12 m}, \quad F_{p}=T \ln \beta \hbar \sqrt{\frac{a}{m}}+\frac{\beta \hbar^{2}(a+\gamma \Gamma)}{24 m} .
$$

At low $T$ and for strong damping one has

$$
T_{x}=\frac{\hbar a}{\pi \gamma} \ln \frac{\gamma^{2}}{a m}, \quad T_{p}=\frac{\hbar \gamma}{\pi m} \ln \frac{\Gamma m}{\gamma}+\frac{\hbar a}{\pi \gamma}, \quad F_{p}=\frac{\hbar \gamma}{2 \pi m} \ln \frac{e \Gamma m}{\gamma}+\frac{\hbar a}{2 \pi \gamma} \ln \frac{\gamma^{2}}{a m} .
$$

The fact that $T_{p} \geq T_{x}>0$ at $T=0$ is related to the quantum nature of the problem.

Generalized thermodynamic formulation. The Wigner function has a quasiGibbsian expression, since there occur two temperature-like variables,

$$
W(p, x)=W_{p}(p) W_{x}(x)=\frac{e^{-p^{2} / 2 m T_{p}}}{\sqrt{2 \pi m T_{p}}} \frac{e^{-a x^{2} / 2 T_{x}}}{\sqrt{2 \pi T_{x} / a}} .
$$

The Boltzmann entropy of momentum and coordinate is

$$
S_{p}=-\int \mathrm{d} p W_{p}(p) \ln \left[W_{p}(p) \sqrt{\hbar}\right]=\frac{1}{2} \ln \frac{e m T_{p}}{\hbar}, \quad S_{x}=\frac{1}{2} \ln \frac{e T_{x}}{\hbar a},
$$


while the von Neumann entropy reads [6] $S_{v N}=\left(v+\frac{1}{2}\right) \ln \left(v+\frac{1}{2}\right)-\left(v-\frac{1}{2}\right) \ln \left(v-\frac{1}{2}\right)$, with $v=\Delta p \Delta x / \hbar=\sqrt{\left\langle p^{2}\right\rangle\left\langle x^{2}\right\rangle} / \hbar=\sqrt{m T_{p} T_{x}} / \hbar \sqrt{a}$. The first terms in its large $v$-expansion, $S_{v N}=\ln v+1$, coincide with the total Boltzmann entropy $S_{B}=S_{p}+S_{x}$.

Internal energy and interaction energy. The energy of the central particle reads $U=$ $\langle\mathscr{H}\rangle=\frac{1}{2} T_{p}+\frac{1}{2} T_{x}$. The energy of the cloud of bath modes that surround the particle is $U_{\text {int }}=U_{\text {tot }}-U_{B}(\gamma=0)-U=U_{p}-U=\Gamma \partial F_{p} / \partial \Gamma$. At high temperatures one has $U=$ $T+\beta \hbar^{2}(2 a+\gamma \Gamma) / 24 m, U_{\text {int }}=\beta \hbar^{2} \gamma \Gamma / 24 m$. Since the energy of the cloud involves $\hbar$, it is a quantum effect. At low temperatures one has, if $\Gamma$ is large, $U_{\text {int }}=\hbar \gamma / 2 \pi m+\mathscr{O}\left(T^{4}\right)$. Implementation of the first law. Given the Hamiltonian $\mathscr{H}(p, x)$ of the subsystem, its energy is $U=\langle\mathscr{H}\rangle=\int \mathrm{d} p \mathrm{~d} x \mathscr{H}(p, x) W(p, x)$, where $W$ is the Wigner function of the subsystem. When we change a system parameter, the energy changes as $\mathrm{d} U=$ $\mathrm{d} \int \mathscr{H} W=\int \mathscr{H} \mathrm{d} W+\int W \mathrm{~d} \mathscr{H} \equiv$ đ $\mathscr{Q}+\mathrm{d} \mathscr{W}$. The first term represents the variation due to the statistical redistribution of the phase space, which we identify with the change in heat đQ2. The last term results from the change in the Hamiltonian, so it is a mechanical, non-statistical object, which we associate with the work đ $\mathscr{W}$ done by external sources.

Generalized free energy and the second law. The definition of the effective temperatures admits a thermodynamical interpretation. The free energy $F$ for a two-temperature system is defined as [13] $F=U-T_{p} S_{p}-T_{x} S_{x}=-\frac{1}{2} T_{p} \ln m T_{p}-\frac{1}{2} T_{x} \ln \left(T_{x} / a\right)$. For adiabatic changes in $m$ or $a$ one has $\mathrm{d} F=-S_{x} \mathrm{~d} T_{x}-S_{p} \mathrm{~d} T_{p}+\mathrm{d} \mathscr{W}$, with work $\mathrm{d} \mathscr{W}=$ $\langle\partial \mathscr{H} / \partial m\rangle \mathrm{d} m+\langle\partial \mathscr{H} / \partial a\rangle \mathrm{d} a=-T_{p} \mathrm{~d} m / 2 m+T_{x} \mathrm{~d} a / 2 a$. Due to the first law this yields the second law for situations with two temperatures, đQ $=T_{p} \mathrm{~d} S_{p}+T_{x} \mathrm{~d} S_{x}$, in close analogy with those proposed recently for nonequilibrium glassy systems [13] and black holes [14]. Notice that $F$ pertains to the particle alone, and does not satisfy Gibbsian thermodynamics, while the Gibbsian $F_{p}$ of Eq. (3), relates to the whole equilibrium system, i.e., to the particle and the cloud of bath modes around it. There are many physical systems, such as a Josephson junction strongly coupled to the electromagnetic field, where the natural object to study is $F$, relating to properties of the junction only.

Violation of the Clausius inequality at low $T$. The Clausius inequality đ $\mathscr{Q} \leq T \mathrm{~d} S_{v N}$ is one of the formulations of the second law. At $T=0$ it says that no heat can be taken from the bath, at best heat can go from the subsystem to the bath.

When we change $a \rightarrow a+\mathrm{d} a$, đQ2 is of order $-T^{2} \mathrm{~d} a$, while $T \mathrm{~d} S_{v N}$ is of order $-T \mathrm{~d} a$. In the case $\mathrm{d} a>0$, where an amount of work $\mathrm{d} \mathscr{W}_{\mathrm{rev}} \sim \mathrm{d} a$ is done on the system, the Clausius relation is thus violated at low $T$. Likewise one can consider the variation of the (effective) mass $m$. Here one has $₫ \mathscr{Q}=\hbar \gamma \mathrm{d} m / 2 \pi m^{2}$. Now there is a transfer of heat even if the bath temperature is zero. Thus, violation of the Clausius inequality is even stronger in this case. This situation with $₫ \mathscr{W}_{\text {rev }}<0$ corresponds to the work performed by the system on the environment. That the heat comes from the cloud of bath modes, is confirmed by the fact that $₫ \mathscr{Q}_{\text {rev }}=-\mathrm{d} U_{\text {int }}$.

Violation of the Landauer bound for information erasure. A further aspect is the squeezing of phase space and entropy, relevant for computing in the quantum regime. We have shown that the so-called Landauer bound $-₫ \mathscr{Q} \geq k T \ln 2$ for the minimal energy dispersion when erasing one bit of information is violated in a similar manner [2]. This connection arises because the Landauer bound is just based on the Clausius inequality.

Dynamics from a non-equilibrium state. Energy oscillation at low $T$. We consider the dynamical evolution of a system initially in equilibrium characterized by a spring 

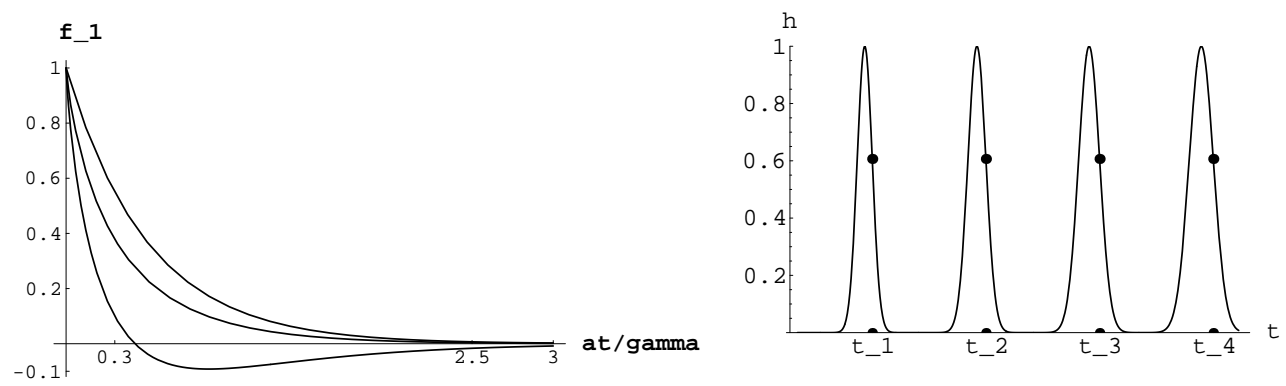

FIGURE 1. a) Normalized excess energy $f_{1}$ as a function of $a t / \gamma$, for different values of the temperature. Upper curve: $T \rightarrow \infty$. Middle curve: $T=\hbar a / 2 \gamma$. Lower curve: at $T=0$ it is non-monotonous. b) Schematic plot of the cyclic changes in the spring constant, where successive cycles are slower and slower. $h$ characterizes the size of the change and $t$ denotes the dimensionless time. The interval $-\infty<t<t_{1}$ marks the process that establishes the nonequilibrium state at $t=t_{1}$. The picture shows three full cycles, in the intervals $t_{i}<t<t_{i+1},(i=1,2,3)$. Their start and end points are indicated by bullets.

constant $a_{0}$, which at $t=0$ is instantaneously changed to $a_{1}=a$. These parameters are connected as $a_{0}=\left(1-\alpha_{0}\right) a$ and we assume that $\left|\alpha_{0}\right| \ll 1$. For strong damping one has for the energy of the subsystem $U(t)=U(\infty)+\left(\hbar a \alpha_{0} / 2 \pi \gamma\right) f_{1}(a t / \gamma)$. The behavior of $f_{1}(\sigma)$ at different temperatures is presented in Fig. 1a. For $\alpha_{0}>0$ it says that, after initially energy has been put on the particle by the change of $a_{0} \rightarrow a>a_{0}$, this energy leaks away into the bath. At low $T$, however, too much leaks away, and a part has to come back. This "bouncing" is familiar of the noise correlator, which behaves like $f_{1}$.

Entropy production. The rate of production of Boltzmann entropy is found after a lengthy identification of its flux. The result can be negative at moderate $T$ [3] . For $T=0$ and strong damping one has with $\sigma=a t / \gamma$ and $\sigma_{\min }=0.879: \mathrm{d}_{i} S_{x} / \mathrm{d} t \sim+\alpha_{0}^{2}(\sigma-$ $\left.\sigma_{\min }\right)\left(\sigma-\sigma_{\min }+\varepsilon\right)$, where $\varepsilon=a m / \gamma^{2}$. This is negative for $\sigma_{\min }-\varepsilon<\sigma<\sigma_{\min }$, or $\Delta t=m / \gamma$, the characteristic timescale of the momentum.

Smooth changes of the spring constant. Let us now consider the case where, starting from the equilibrium state $a(-\infty)=a$, the spring constant $a(t)=[1-\alpha(t)] a$, is slightly changed $(|\alpha(t)| \ll 1)$ in a smooth way. The rate of work added to the system is $\mathrm{d} \mathscr{W} / \mathrm{d} t=$ $\mathrm{d} \mathscr{W}_{\text {rev }} / \mathrm{d} t+\mathrm{d} \Pi / \mathrm{d} t$, with the adiabatic ('reversible') and dispersed contributions

$$
\frac{\mathrm{d} \mathscr{W}_{\mathrm{rev}}}{\mathrm{d} t}=-\frac{\gamma \alpha^{\prime}(\tau)}{4 m}\left[T_{x}+\frac{\hbar a}{\pi \gamma} \alpha(\tau) C_{x}(0)\right], \quad \frac{\mathrm{d} \Pi}{\mathrm{d} t}=\frac{\hbar a \alpha^{\prime}(\tau)}{4 \pi m} \int_{0}^{\infty} \mathrm{d} \sigma \alpha^{\prime}(\tau-\sigma) C_{x}(\sigma) .
$$

where $\tau=\gamma t / 2 m$ and $C_{x}$ a function like $f_{1}$ of Fig. 1a. Integrating over the full change (the whole region where $\alpha^{\prime} \neq 0$ ) one confirms that the dispersion for a completed, cyclic change $\left(\alpha_{i}=\alpha_{f}\right)$ of system parameters is nonnegative, the Thomson formulation of the second law [15]. It is also nonnegative for noncyclic $\left(\alpha_{i} \neq \alpha_{f}\right)$ but completed changes.

Energy dispersion. If $\alpha(\tau)=\alpha_{m} h(\Omega t)$, with $\Omega$ a slow rate of change, and $\gamma$ is large, expansion in $\Omega$ is possible. Omitting numerical factors one gets the structure

$$
\frac{\mathrm{d} \Pi}{\mathrm{d} t}=\hbar \alpha_{m}^{2} \Omega^{2} h^{\prime}\left[\left(\frac{\gamma T}{\hbar a}\right)^{2} h^{\prime}+\frac{\gamma \Omega}{a} h^{\prime \prime}-\frac{\gamma^{2} \Omega^{2}}{a^{2}} h^{\prime \prime \prime}\right] .
$$


The full integral is always positive and for moderate $T$ the first term is dominant. However, for small $T$ the term $h^{\prime} h^{\prime \prime}$ may imply that $\mathrm{d} \Pi / \mathrm{d} t$ is negative. This is a firm statement, also valid when starting in equilibrium, since work is the energy added to the total system. It is the more surprising since the relevant domain the characteristic timescale of the change of $a, 1 / \Omega<\hbar^{2} /\left(\gamma T^{2}\right)$, may exceed all other timescales, including the quantum timescale $\hbar / T$, so that applicability of thermodynamics was to be expected.

Perpetuum mobile with many work extraction cycles. The $h^{\prime} h^{\prime \prime}$ term can cause extraction of work. Let there be $\mathscr{N}$ non-overlapping Gaussians, $h(x)=\exp \left(-\frac{1}{2} x^{2}\right)$, as depicted in Figure 1b. Cycles are the intervals $t_{i}<t<t_{i+1}(i \geq 1)$. Each new cycle is slower than the previous one, $\Omega_{n+1}<\Omega_{n}$. One can make a total number $\mathscr{N} \sim 1 / T$ of cycles with equal yield, where $\mathscr{N}$ is parameterized by $v$, and total yield

$$
\mathscr{N}=\frac{\hbar a}{2 \pi \gamma T} \int_{\beta \hbar \Omega_{\mathscr{N}} / 2 \pi}^{\beta \hbar \Omega_{1} / 2 \pi} \frac{\mathrm{d} y y}{v+I_{1} y+I_{3} y^{3}}, \quad \mathscr{W}_{\mathrm{tot}}=-\frac{\pi \alpha_{\mathrm{m}}^{2}}{3} \frac{\gamma T^{2}}{\hbar a} \int_{\beta \hbar \Omega_{\mathscr{N}} / 2 \pi}^{\beta \hbar \Omega_{1} / 2 \pi} \mathrm{d} y \frac{v y}{v+I_{1} y+I_{3} y^{3}} .
$$

with $I_{1}=\frac{1}{2} \sqrt{\pi}, I_{3}=3 \sqrt{\pi} / 4$. The minus sign of $\mathscr{W}_{\text {tot }}$ indicates that work is performed by the system on the environment. This is possible because, in order to make the extraction cycles, one had to start from the equilibrium state $\alpha(-\infty)=0$ and change $\alpha$ up to $\alpha\left(t_{1}\right)$. In this first part the energy dispersion was $\Pi\left(t_{1}\right)=+\alpha_{\mathrm{m}}^{2} \hbar \gamma \Omega_{1}^{2} / 24 \pi a>\left|\mathscr{W}_{\text {tot }}\right|$.

Feasibility. The harmonic oscillator can be interpreted as an LC circuit [16]. $x$ may correspond to the charge $Q$ on a capacitor, $1 / a$ to its capacitance $C, m$ to an inductance $L, p$ to a phase $\Phi, \gamma$ to a resistance $R$, and $\eta(t)$ to a random electro-motoric force. In this setup there should be nothing difficult in varying $L \sim m$ or $C \sim 1 / a$. We have proposed to test the violation of the Clausius inequality in mesocopic linear circuits [17]. This amounts to measuring $\left\langle\delta Q^{2}\right\rangle,\left\langle\delta \Phi^{2}\right\rangle$ and the produced work. The conditions to do this experiment were reached 20 years ago, and one of the quantities, $\left\langle\delta Q^{2}\right\rangle$, was measured already and agrees perfectly with the theoretical predictions [18, 19].

Has thermodynamics been violated? Let us recall that our results hold also for $N$ non-interacting Brownian particles in a bath. Our conclusion is that thermodynamics does not always work when, in the quantum regime, one considers the Brownian particle in its reduced Hilbert space, thus summing out the bath. We should admit that at low enough $T$ the interaction energy between system and bath becomes relevant, since the damping constant is fixed. In a very strict definition of thermodynamics one may claim that there is no reason why thermodynamics had to apply.

For this reason our surprising findings of the breakdown of the Clausius inequality can in principle be viewed as results outside the domain of applicability of thermodynamics. However, the negativity of the Boltzmann entropy production sets in already at moderate $T$, more or less in the same domain where energy initially put on the particle starts to dissipate to the bath in a non-monotonic fashion, even in limit of large damping.

Our second effect in that regime, the presence of many work extraction cycles ("perpetuum mobile") involves only the energy budget of the total system, and should be more surprising. However, when starting in an equilibrium state and counting all the energy, the total dispersion is positive, as demanded by an exact theorem [15].

These extraction cycles relied on the fact that the rate of energy dispersion by the total system can be negative. This is a firm and unexpected statement about the total system, that may have started in equilibrium. Thus positivity of the rate of energy dispersion 
[20] is not always a good formulation of the second law.

The new aspects of quantum Brownian motion arise from quantum entanglement: A complete description in terms of a wave function is possible only for a closed system; subsystems are necessarily in a mixed state. Thus the quantum Gibbs distribution is not an adequate candidate for the description of the quantum subsystem non-weakly interacting with its thermal bath. Connections of these findings with NMR physics [21], mesoscopic work sources [15] and with Maxwell's demon [22] are discussed elsewhere.

Acknowledgments. We thank R. Balian and L.S. Suttorp for discussion.

\section{REFERENCES}

1. L.D. Landau and E.M. Lifshitz, Statistical Physics, Part 1, (Pergamon, London, 1980).

2. A.E. Allahverdyan and Th.M. Nieuwenhuizen, Extracting work from a single thermal bath in the quantum regime, Phys. Rev. Lett. 85 (2000) 1799; Breakdown of the Landauer bound for information erasure in the quantum regime, Phys. Rev. E 64, 056117 (2001).

3. Th.M. Nieuwenhuizen and A.E. Allahverdyan, Statistical thermodynamics of quantum Brownian motion: Construction of perpetuum mobile of the second kind, Phys. Rev. E (2002); cond-mat/0011389.

4. Philip F. Schewe and Ben Stein, New frontiers of thermodynamics, http://www.aip.org/enews physnews/2000/split/pnu494-1.htm; Peter Weiss, Breaking the law: Can quantum mechanics + thermodynamics = perpetual motion ?, Sci. News (Washington D.C.)158 234 (2000).

5. Yu. L. Klimontovich, Statistical Theory of Open Systems, (Kluwer, Amsterdam, 1997).

6. U. Weiss, Quantum Dissipative Systems, (World Scientific, Singapore, 1993).

7. H. Risken, The Fokker-Planck Equation, (Springer, Berlin, 1984).

8. A.O. Caldeira and A.J. Leggett, Ann. Phys. 149, 374, (1983).

9. C.W. Gardiner, Quantum Noise, (Springer-Verlag, 1991).

10. R.H. Koch, D.J. Van Harlingen and J. Clarke, Phys. Rev. Lett. 45, 2132, (1980); ibid 47, 1216 (1981).

11. K.K. Likharev, Sov. Phys. Usp. 26, 87 (1983).

12. K. Suominen, B. M. Garraway, and S. Stenholm, Phys. Rev. A 453060 (1992).

13. Th.M. Nieuwenhuizen, Thermodynamic description of a dynamical glassy transition, J. Phys. A 31, L201, (1998); Thermodynamics of the glassy state: effective temperature as an additional system parameter, Phys. Rev. Lett. 805580 (1998); Thermodynamic picture of the glassy state gained from exactly solvable models, Phys. Rev. E 61267 (2000); Formulation of thermodynamics for the glassy state: configurational energy as a modest source of energy, J. Chem. Phys. 1158083 (2001); A.E. Allahverdyan and Th.M. Nieuwenhuizen, Entropy production, energy dissipation and violation of Onsager relations in the steady adiabatic state, Phys. Rev. E 62845 (2000).

14. Th.M. Nieuwenhuizen, Thermodynamics of black holes: an analogy with glasses, Phys. Rev. Lett. 812201 (1998).

15. A.E. Allahverdyan, R. Balian and Th.M. Nieuwenhuizen, Thomson's formulation of the second law: an exact theorem and limits of its validity, these proceedings and preprint 2002; A.E. Allahverdyan and Th.M. Nieuwenhuizen, A mathematical theorem as the basis for the second law: Thomson's formulation applied to equilibrium, Physica A 305, 542 (2002).

16. Yu. L. Klimontovich, Sov. Phys. Usp. 30, 154 (1987).

17. A.E. Allahverdyan and Th.M. Nieuwenhuizen, On testing the violation of the Clausius inequality in nanoscale electric circuits, Phys. Rev. B, to appear; cond-mat/0205156.

18. A.N. Cleland, J.M. Schmidt and J. Clarke, Phys. Rev. B 45, 2950, (1992).

19. G.Y. Hu and R.F. O‘Connell, Phys. Rev. B 46, 14 219, (1992).

20. P. Kondopudi and I. Prigogine, Modern thermodynamics: from heat engines to dissipative structures, (Wiley, West Sussex, UK, 1998).

21. C. Pombo, A.E. Allahverdyan and Th.M. Nieuwenhuizen, Bath generated work extraction in twolevel systems these proceedings; A.E. Allahverdyan and Th.M. Nieuwenhuizen, Bath generated work extraction and inversion-free gain in two-level systems, cond-mat/0201408.

22. Th.M. Nieuwenhuizen and A.E. Allahverdyan, Unmasking Maxwell's Demon, these proceedings. 